\title{
Confiança interorganizacional e cooperação em habitats de inovação
}

\section{Nádia Carvalho}

Professora do Departamento de Administração da Universidade Federal de Juiz de Fora- Campus Governador Valadares. Doutoranda em Administração na Universidade Federal do Espírito Santo. Mestre e graduada em Administração pela Universidade Federal de Lavras, Minas Gerais, Brasil

nadia.carvalho@ufjf.edu.br

Hélio Zanquetto Filho

Professor do Departamento de Administração da Universidade Federal do Espírito Santo. Doutor e mestre em Engenharia de Produção pela Pontifícia Universidade Católica do Rio de Janeiro. Graduado em Engenharia Civil pela Universidade Federal do Espírito Santo, Espirito Santo, Brasil

zanquetto@gmail.com

Marcos Paulo Valadares de Oliveira

Professor do Departamento de Administração da Universidade Federal do Espírito Santo. Visiting scholar na North Carolina State University. Doutor, mestre e graduado em Administração pela Universidade Federal de Minas Gerais, Espirito Santo, Brasil marcos.p.oliveira@ufes.br

\section{Editor Científico: José Edson Lara}

Organização Comitê Científico

Double Blind Review pelo SEER/OJS

Recebido em 17.07.2017

Aprovado em 25.01.2018 


\title{
Resumo
}

Ao considerar o modelo de inovação aberta, diante da cooperação entre agentes de inovação, a confiança é vista como um de seus principais fatores. Neste sentido, este estudo buscou investigar alguns fatores formadores da confiança interorganizacional, bem como seu impacto na cooperação de empresas pertencentes a habitats de inovação. Trata-se de uma pesquisa quantitativa, tendo como técnica de coleta de dados o survey; para a análise dos dados foi utilizada a técnica da modelagem de equações estruturais. A amostra foi composta por 68 empresas pertencentes a espaços de inovação vinculados à Rede Mineira de Inovação (RMI). Os resultados indicaram que a formação da confiança interorganizacional leva em consideração a reputação, boa vontade, a confiança interpessoal, competência e o capital social. A confiança exerce um alto impacto positivo na cooperação, ao contrário do oportunismo, que modera a relação impactando negativamente.

Palavras-chave: Confiança interorganizacional; Cooperação; Equações Estruturais; Habitats de Inovação.

\section{Interorganizational trust and cooperation in innovation habitats}

\begin{abstract}
Considering the open innovation model, in the face of cooperation between innovation agents, trust is one of the main factors. In this sense, this study sought to investigate some factors that form interorganizational trust, as well as its impact on the cooperation of companies belonging to innovation habitats. It is a quantitative research, having as data collection technique the survey and for the analysis of the data was used the technique of structural equation modeling. The sample was composed by 68 companies belonging to innovation spaces linked to the Rede Mineira de Inovação (RMI). The results indicated that the formation of interorganizational trust considers the reputation, good will, interpersonal trust, competence and social capital. Trust has a high positive impact in the cooperation, as opposed to the opportunism that moderates the relation negatively impacting.
\end{abstract}

Keywords: Interorganizational trust; Cooperation; Structural Equations; Innovation Habitats.

\section{Confianza interorganizacional y cooperación en los hábitats de la innovación}

\section{Resumen}

Considerando el modelo de innovación abierta, ante la cooperación entre agentes de innovación, la confianza es uno de los principales factores. En este sentido, este estudio buscó investigar algunos factores formadores de la confianza 
interorganizacional, así como su impacto en la cooperación de empresas pertenecientes a habitats de innovación. Se trata de una investigación cuantitativa, teniendo como técnica de recolección de datos el survey y para el análisis de los datos se utilizó la técnica del modelado de ecuaciones estructurales. La muestra fue compuesta por 68 empresas pertenecientes a espacios de innovación vinculados a la Red Minera de Innovación (RMI). Los resultados indican que la formación de la confianza interorganizacional tiene en cuenta la reputación, la buena voluntad, la confianza interpersonal, la competencia y el capital social. La confianza ejerce un alto impacto positivo en la cooperación, a diferencia del oportunismo que modifica la relación impactando negativamente.

Palabras clave: Confianza interorganizacional; Cooperación; Ecuaciones Estructurales; Hábitats de Innovación.

\section{Introdução}

No século XX o processo de inovação era pautado por um modelo fechado, em que desde a geração de ideias até seu desenvolvimento, comercialização, financiamento e outras etapas possuíam foco interno. Porém, no final do século XX alguns fatores como mobilidade de profissionais, presença de capital privado de risco, competitividade, dentre outros, fizeram com que este modelo não mais fosse sustentável. Ao romper com o modelo fechado, emerge o modelo denominado inovação aberta (open innovation) (Chesbrough, 2012). Assim, o modelo de inovação aberta aponta a oportunidade de as empresas realizarem atividades de Pesquisa \& Desenvolvimento (P\&D) de forma mais interativa, buscando a integração de conhecimentos.

Nesse sentido, a inovação aberta estimula a cooperação, desenvolvendo um papel essencial na aproximação dos agentes de inovação, uma vez que essa relação permite a integração de conhecimento e sua transferência, além da geração conjunta de soluções (Forcadell \& Guadamillas, 2002; Lavie \& Drori, 2012). Sendo assim, a cooperação desempenha um papel-chave na construção de verdadeiros reservatórios de conhecimento. Este fenômeno se desenvolve por meio de relações fora dos limites organizacionais, fazendo com que todo o conhecimento compartilhado se torne parte do conhecimento total da organização (Parashar, 2007).

Melo (2010) afirma que o compartilhamento de informações e conhecimento pode ocorrer por meio dos habitats de inovação $(\mathrm{HI})$, caracterizados como espaços relacionais que permitem o processo de aprendizagem mediante as interações. 
Portanto, habitat de inovação refere-se ao ambiente físico de inovação que proporciona suporte aos empreendedores, ou seja, é o espaço em que ideias são transformadas em empreendimentos de sucesso (Machado, Silva, \& Catapan 2016). Tais espaços podem ser chamados de Núcleos de Inovação Tecnológica, Centros de Inovação, Parques Tecnológicos, Incubadoras e Polos Tecnológicos (Luz et al., 2014).

Para tanto, Bukowitz e Williams (2002) ressaltam que compartilhar conhecimento envolve motivação, facilitação e confiança. Brattstrom, Lofsten e Richtnér (2012) consideram a confiança um elemento importante para inovação, pois permite melhorar o processo de aprendizagem, bem como estimular a criatividade, além de ser importante para o sucesso das parcerias e alianças estratégicas estabelecidas (Ali Babar, Verner, \& Nguyen, 2007). Portanto, fatores como a adaptação, relacionamento de longo prazo, interdependência e confiança mútua são importantes para o desenvolvimento de inovações (Halli, Holm, \& Sharma, 2011). Sendo assim, presume-se que a confiança interorganizacional influencia a cooperação entre as organizações que buscam desenvolver o modelo de inovação aberta.

Confiança, para Fukuyama (1996), refere-se à expectativa a partir de um comportamento estável, honesto e cooperativo. Para Das e Teng (2001) a confiança interorganizacional reflete esta definição, na medida em que é compreendida como a expectativa positiva de uma organização em relação a outra organização.

Assim, visando o modelo de inovação aberta, em que impera a integração de agentes econômicos de inovação, a confiança se insere como um dos fatores determinantes no que se refere ao sucesso da cooperação (Thorgren \& Wincent, 2011). Neste mesmo sentido, Chesbrough, Sohyeong e Agogino (2014) concluíram que o sucesso empresarial depende da confiança entre as partes envolvidas no ecossistema de inovação ao qual pertencem.

Apesar de ser tema de diversos estudos, a confiança é tida como um fator multidimensional, o que dificulta uma definição única e, ao mesmo tempo, compreender seus elementos torna-se um desafio (Seppanen, Blomqvist, \& Sundqvist, 2007). Assim, a confiança é uma variável ainda não completamente definida, o que pode ser considerado natural para um fenômeno complexo 
(Bhattachary, Timothy, \& Madan, 1998). Deste modo, tornam-se relevantes estudos que visam a identificar e compreender as relações dos elementos da confiança.

Portanto, o objetivo deste estudo é identificar fatores da formação da confiança interorganizacional, bem como seu impacto na cooperação para empresas pertencentes a habitats de inovação. Dessa forma, como contribuição teóricoempírica, este estudo baseia-se tanto na lacuna de compreensão dos elementos formadores da confiança, bem como na carência de estudos que associam confiança à inovação, conforme Zimmer, Luz Filho e Bloemer (2015). Assim, esperase contribuir para ampliar o campo empírico sobre o tema além de oferecer contribuições de ordem prática, uma vez que os resultados encontrados podem colaborar com elementos para fomentar a cooperação entre as empresas.

\section{Revisão de Literatura}

Difundido por Chesbrough (2003), o modelo de inovação aberta surgiu como novo paradigma que explora a difusão do conhecimento. Sendo uma alternativa estratégica importante para as empresas obterem vantagem competitiva (Drechsler \& Natter, 2012), o modelo é conceituado diante dos fluxos propositais de entrada e saída de conhecimento para acelerar a inovação interna e expandir os mercados para o uso externo de inovação, respectivamente (Chesbrough 2006).

Dessa forma, a inovação aberta envolve um processo evolutivo de aprendizagem coletiva em que diferentes partes podem se unir e cooperar, como por exemplo as empresas, as instituições de pesquisa, clientes, governos, instituições financeiras, e outras (Van Mierlo, Leeuwis, Smits, \& Woolthuis, 2010).

Neste contexto, os relacionamentos sociais concebem uma alternativa de acesso a novos recursos; tais relacionamentos tanto podem ser informais, baseado em confiança, ou formais, baseados em contratos (Cunha, Passador, \& Passador, 2012).

Por confiança, Fukuyama (1996) entende que se refere à expectativa a partir de um comportamento estável, honesto e cooperativo. Sendo assim, a confiança interorganizacional reflete esta definição, na medida em que é compreendida como a expectativa positiva de uma organização em relação a outra organização (Das \& Teng, 2001; Morgan \& Hunt, 1994; Rousseau, Sitkin, Burt, \& Camerer, 1998). Dessa forma, Zaheer, Mcevily e Perrone (1998) definem confiança interorganizacional como 
aquela atribuída ao conjunto de relações estabelecidas de uma organização com outra.

Confiança é uma variável ainda não completamente definida na literatura (Das \& Teng, 2001; Newell \& Swan, 2000; Zarvandi \& Zarvandi, 2012), sendo objeto de estudo em diferentes contextos (Bhattacharya, Timothy, \& Madan, 1998). Assim, Fink, Kessler, Duh, Belak e Lang (2009) identificaram que a confiança foi o fator que mais contribuiu para a explicação do desempenho financeiro de pequenas e médias empresas envolvidas em relacionamentos interorganizacionais. Estudos também demonstram os benefícios da confiança em acordos de cooperação, como a redução dos custos de transação, mitigação do risco de investimento em ativos específicos e facilitação do processo de tomada de decisão (Thorgren \& Wincent, 2011).

No que se refere à cooperação interorganizacional, esta se relaciona com a iniciativa e a participação em acordos colaborativos entre organizações, o que permite acesso a clientes, recursos e capacidades complementares dos parceiros, aprendizado e acúmulo de tecnologia (Hao, 2004). Dessa forma, com a necessidade crescente de conhecimentos científicos pelas empresas, práticas de cooperação tecnológica são cada vez mais exigidas entre os atores envolvidos no processo de geração, manutenção e difusão de inovações (Abdalla, Calvosa, \& Batista, 2009).

Para Britto (2004) pode-se identificar como impacto da consolidação da cooperação sua dimensão dupla do processo, sendo ao mesmo tempo um instrumento eficaz de processamento de informação; também constitui uma alternativa para viabilizar a aglutinação de competências complementares; permite um melhor enfrentamento da turbulência ambiental e facilita a identificação e exploração de novas oportunidades. Dessa forma, a cooperação admite uma maior comunicação entre os agentes, o que permite a integração das competências, além de consolidar a confiança mútua dos agentes e uma maior sincronia nas ações estratégicas adotadas por eles.

\subsection{Delimitação dos Construtos}

Para Das e Teng (2001) e Nooteboom (1996) confiança pode ser compreendida a partir de duas óticas: confiança baseada na competência (competence trust), em que se baseia nas habilidades do parceiro de ter 
desempenho conforme o acordado e confiança baseada na intenção, ou boa vontade (goodwill trust).

\subsubsection{Formação da Confiança Interorganizacional}

A confiança baseada em competência reflete a confiança de uma empresa na capacidade de um parceiro de cumprir com a obrigação acordada (Lui \& Ngo, 2004). Assim, a formação de uma parceria baseia-se geralmente no pressuposto de que os futuros parceiros possuem competência adequada no que se refere ao conhecimento, habilidades e capacidades necessárias para cumprir os objetivos planejados (Malhotra \& Lumineau, 2011). Portanto, a confiança na competência, embora tenha recebido muito menos atenção empírica do que a confiança da boa vontade, também é essencial para alianças (Wittmann, Hunt, \& Arnett, 2009).

Jiang, Li, Gao, Bao e Jiang (2013) afirmam que, para empresas que buscam a cooperação, a competência é a dimensão de maior importância em níveis elevados de contratos, o que tende a minimizar o vazamento de informações e, portanto, o risco. Dessa forma, espera-se que a formação da confiança interorganizacional se dê mediante o construto competência, uma vez que o presente estudo assume que a confiança baseada na competência refere-se à capacidade e habilidade do parceiro de ter desempenho conforme o acordado (Das \& Teng, 2001; Nooteboom, 1996). Assim, pretendeu-se testar empiricamente a seguinte hipótese:

\section{H1: A competência do parceiro forma a confiança interorganizacional.}

Já a confiança baseada na intenção ou boa vontade (goodwill trust) é emocional, com base na benevolência, integridade, na boa-fé e se preocupa com o bem-estar da outra parte (Das \& Teng, 1998; Das \& Teng, 2001; Lewis \& Weigert, 1985). Portanto, quando a confiança de boa vontade está presente, há uma cooperação mais estreita, um intercâmbio de informações mais aberto, um maior grau de comprometimento e uma maior interação informal entre as partes (Fryxell, Robert, \& Vryza, 2002; Lui \& Ngo, 2004).

Em um nível individual, Shazi, Gillespie e Steen (2015) examinaram a confiança na formação de laços e redes sociais voltadas para inovação e os resultados demonstram que as relações desenvolvidas foram pautadas pela 
habilidade e benevolência. Assim, sugerem que as pessoas precisam perceber os outros como benevolentes, ou seja, com boa vontade, a fim de buscarem suas habilidades e conhecimentos complementares para a inovação.

Dessa forma, espera-se também que a formação da confiança interorganizacional perpasse o construto boa vontade, que é assumido neste estudo como a crença na integridade das organizações parceiras, ou seja, na intenção positiva no momento em que se acredita no outro, a partir do que foi estabelecido (Das \& Teng, 2001; Nooteboom, 1996). Assim, também pretendeu-se testar empiricamente a hipótese:

\section{H2: A boa vontade do parceiro forma a confiança interorganizacional.}

Além desses fatores, existe ainda uma distinção muito tênue entre confiança interorganizacional e confiança interpessoal, pois as duas estão presentes nas organizações. Sol, Beers e Wals (2013), ao analisarem o processo de aprendizado em redes de inovação, identificaram que a confiança é produzida diante das relações pessoais dos atores.

Nesse sentido, Barney e Hansen (1994) afirmam que a confiança exerce uma importante influência na concretização dos relacionamentos interorganizacionais cooperativos e que esta depende principalmente da confiança interpessoal. Tacconi, Lopes, Mol, \& Tacconi Neto (2014) consideram confiança iertorganizacional e interpessoal construtos distintos porém imbricados em um mesmo sistema de relações. Portanto, assumindo que confiança interpessoal é definida como a confiança de um agente organizacional que é depositada no agente de outra organização, ou seja, dentro do limite individual (Zaheer et al.,1998), emerge a terceira hipótese:

\section{H3: A confiança interpessoal forma a confiança interorganizacional.}

Estudos anteriores mostraram que a reputação do parceiro é importante no processo de cooperação (Del Campo, Pardo, \& Perlines, 2014), sendo que uma reputação positiva pode reduzir as assimetrias de informação (Houston, 2003), tornando-se extremamente importante para a confiança entre os parceiros. Assim, 
as empresas buscam parceiros com boa reputação, uma vez que essa reputação transmite confiança durante as fases iniciais de um acordo e, portanto, leva a um início efetivo da relação (Das \& Teng, 1998).

Portanto, no presente estudo entende-se que a reputação se refere à integridade da organização parceira. Neste sentido, a integridade se relaciona com as características comportamentais, ou seja, pela idoneidade da organização parceira (Mcallister, 1995). Portanto, tem-se a quarta hipótese:

\section{H4: A Reputação do parceiro forma a confiança interorganizacional.}

Outra dimensão importante na discussão da confiança interorganizacional é sobre o capital social, ou seja, as relações estabelecidas que permitem acesso a recursos. O capital social se refere à soma dos recursos envolvidos a partir da rede de relacionamentos (Nahapiet \& Ghoshal, 1998). Nesse sentido, os autores definiram o capital social a partir de três dimensões distintas e inter-relacionadas, considerando a integração de suas diferentes facetas. Dessa forma, consideram a dimensão estrutural, que versa sobre as conexões entre os atores; a dimensão relacional, que se refere aos ativos criados e impulsionados por imersões em relacionamentos específicos; e a dimensão cognitiva, referente aos recursos que fornecem representações, interpretações e sistemas de significações compartilhados.

As dimensões discutidas por Nahapiet e Ghoshal (1998) evidenciam uma abordagem que facilita a cooperação; dessa forma, as dimensões são articuladas promovendo o capital social e se relacionando com a confiança, pois permite às organizações acessarem conhecimentos e recursos externos, o que para Anand, Glick e Manz (2002) contribui para uma melhoria da eficácia dos processos decisórios, absorção de inovações em produtos e processos e a conquista de novos mercados.

Portanto, entende-se, que capital social versa sobre os recursos que as organizações obtêm por meio de suas relações com outras organizações (Zahra, 2010), o que permite uma criação de valor e aprendizagem pelas organizações (Czakon, 2009). A partir dessas relações, é que pretendeu-se testar empiricamente a seguinte hipótese: 


\section{H5: O Capital Social forma a Confiança Interorganizacional.}

Assim, corroborando a argumentação de Rousseau et al. (1998), que sustentam que é possível classificar a confiança em dimensões que se referem ao seu conteúdo, após a revisão acima apresentada, no que se refere à delimitação da confiança interorganizacional, o presente trabalho entende que esta é formada pelas dimensões Competência, Reputação, Boa Vontade, Capital Social e Confiança Interpessoal, conforme apresentado no modelo de pesquisa proposto na Figura 1.

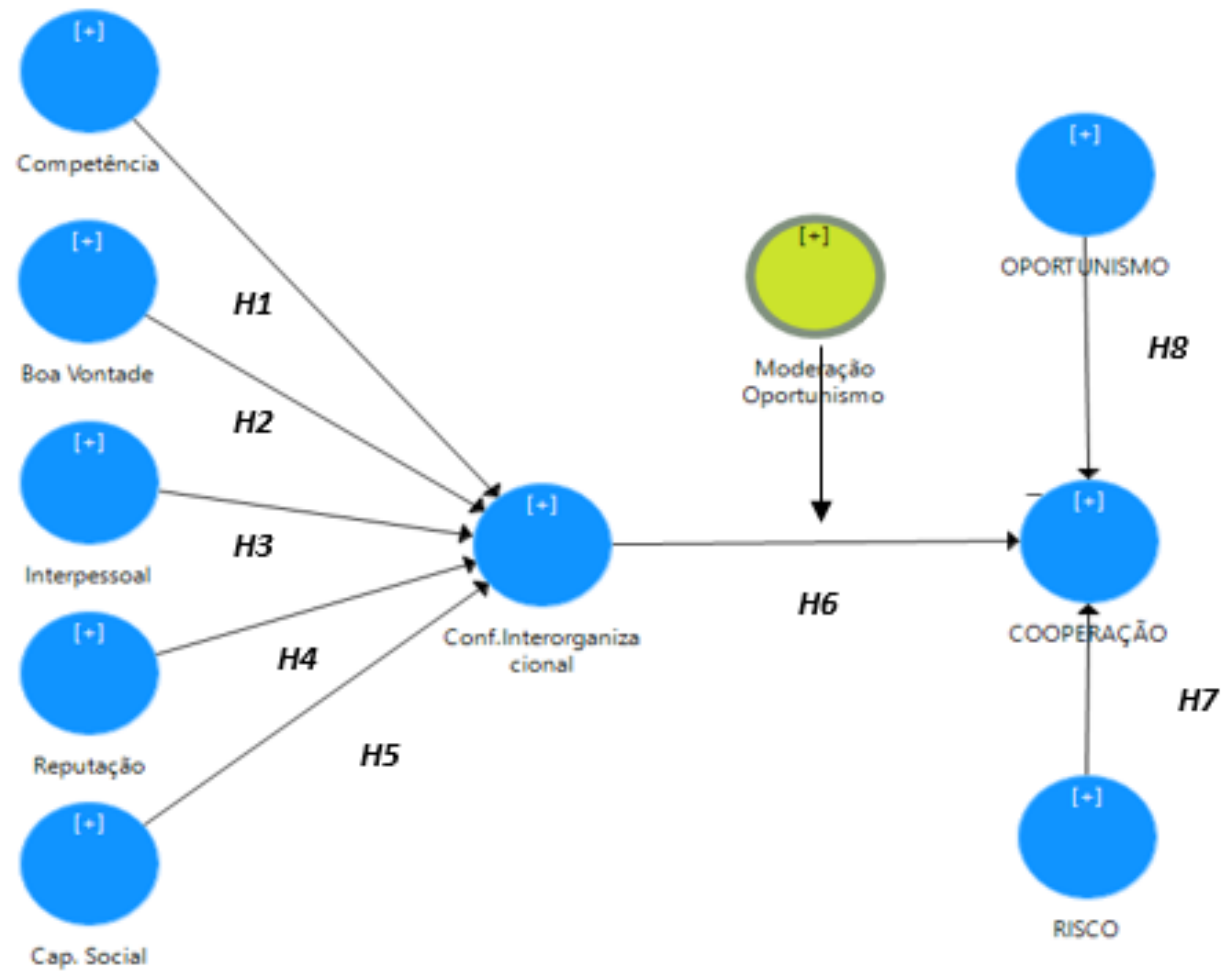

\section{Figura 1}

Modelo com Hipóteses propostas

Fonte: Elaborado pelos autores.

\subsection{Relacionamento entre os construtos e efeito moderador}

A confiança é apresentada como um dos fatores mais determinantes no sucesso da cooperação (Del Campo, Pardo, \& Perlines, 2014), pois permite reduzir o risco e a incerteza e a necessidade de controle em situações empreendedoras complexas (Gulati \& Higgins, 2003; Stuart, 2000; Thorgren \& Wincent, 2011). Além 
disso, Hallin, Holm e Sharma (2011) argumentam que fatores como a adaptação, relacionamento de longo prazo, interdependência e confiança mútua são importantes para o desenvolvimento de inovações.

Dessa forma, um alto nível de confiança permite que os parceiros compartilhem conhecimento, recursos e capacidades, o que tem um impacto positivo nos resultados do acordo, já que os parceiros se sentem seguros contra comportamentos oportunistas uns com os outros (Stuart, 2000).

Para a pesquisa, assume-se a cooperação como a iniciativa e a participação em acordos colaborativos com outras organizações, o que permite acesso a clientes, recursos e capacidades complementares dos parceiros, aprendizado e acúmulo de tecnologia, além da produção em escopo ou escala, ou seja, é o resultado da ação conjunta de diferentes atores (Hao, 2004; Porto, 2004). Portanto, diante do exposto, tem-se a seguinte hipótese:

\section{H6: Confiança Interorganizacional impacta a cooperação entre empresas.}

Porém, ao tratar dos construtos confiança e cooperação, dois outros construtos são importantes para a relação: o oportunismo e o risco. Johnston, Mccutcheon, Stuart e Kerwood (2004) consideram que a confiança não é simplesmente um input para o relacionamento, mas também um pré-requisito e uma consequência, em que a incerteza é uma condição sine qua non da confiança (Hald, Cordon, \& Vollmann, 2009), ou seja, aceitação de risco é uma das principais consequências da confiança (Luhmann, 2000).

Sendo assim, embora as alianças estratégicas ofereçam oportunidades para o compartilhamento de conhecimento, também levam ao risco, uma vez que pode haver vazamento de conhecimento para as empresas parceiras (Jiang et al., 2013).

Portanto, a confiança se relaciona com o risco, no sentido em que se caracteriza pela incerteza de resultados futuros (Evans \& Olsson, 2002), e por risco entende-se, na pesquisa, como a probabilidade de perda (Diniz \& Marconatto, 2010). Assim, espera-se que o risco se relacione com a cooperação mediante a confiança, e neste sentido buscou-se testar a seguinte hipótese: 


\section{H7: O risco impacta a cooperação a partir da confiança estabelecida.}

Nesta mesma linha, o oportunismo também se destaca na relação da confiança e cooperação, uma vez que ao possuir confiança esta evitaria comportamentos oportunistas na colaboração entre empresas (Laaksonen, Jarimo, \& Kulmala, 2009). Por oportunismo se entende a busca pelo auto interesse. Dessa forma, por ser impossível total controle do parceiro, uma das partes fica vulnerável às ações da outra parte, com a expectativa de honestidade do parceiro (Cunha \& Melo, 2006; Das \& Teng, 1998; Mayer, Davis, \& Schoorman, 1995). Portanto, o oportunismo insere um componente de incerteza nas relações, uma vez que não há como prever exatamente o comportamento dos agentes (Cabral, 2008). Neste sentido, pretendeu-se testar empiricamente a hipótese:

\section{H8: O oportunismo modera a relação confiança e cooperação.}

\section{Procedimentos Metodológicos}

A pesquisa é do tipo quantitativa, referindo-se a dados e informações acerca de opiniões e características de um grupo determinado, a partir de uma população alvo (Freitas, Oliveira, Saccol, \& Moscarola, 2000). Dessa forma, foi utilizado o método survey para coleta de dados que foi aplicado em empresas pertencentes a habitats de inovação. Para tanto, a amostra da pesquisa foi delimitada aos habitats associados à Rede Mineira de Inovação (RMI), que possui como objetivo a integração da pesquisa, tecnologia e inovação, visando a gerar negócios competitivos (RMI, 2016). Atualmente a RMI possui 25 incubadoras, 3 parques tecnológicos e 2 outras instituições, uma de pesquisa e outra de ensino como associados. A escolha do recorte se deu diante da possibilidade de acesso aos gestores das incubadoras e parques, totalizando 28 espaços vinculados à RMI.

Assim, após a elaboração do instrumento de pesquisa, o mesmo foi valiado conceitualmente por um pesquisador da área e na sequência por um gestor de um habitat de inovação não pertencente à amostra da pesquisa. Após alguns ajustes, o questionário foi então enviado aos gestores dos 28 espaços associados à RMI, utilizando a plataforma SurveyMonkey. Portanto, foi solicitado que o questionário 
fosse encaminhado aos gestores das empresas pertencentes aos habitats. Em média, esses espaços somam cerca de 200 empresas e, ao todo, foram retornados 75 questionários, sendo que, após análise dos mesmos, 7 foram descartados por estarem incompletos, sendo obtidos 68 questionários válidos. A aplicação do questionário ocorreu no segundo semestre de 2016, sendo os respondentes convidados a assinalarem suas percepções sobre os aspectos relacionados à confiança e cooperação em uma escala de Likert de 7 pontos.

A Tabela 1 em apêndice apresenta a operacionalização das variáveis utilizadas no modelo. A validade conceitual dos construtos foi desenvolvida a partir da revisão de literatura e, dessa forma, as escalas utilizadas foram baseadas na revisão conceitual dos construtos.

Vale ressaltar que o construto oportunismo foi medido como não oportunismo, ou seja, com uma escala positiva, uma vez que objetivou seguir as demais para não confundir o respondente. Sendo assim, para a análise dos resultados foi necessária a inversão da escala do oportunismo.

Objetivando verificar a formação da Confiança Interorganizacional e seu impacto na Cooperação, os dados coletados foram tratados diante da técnica multivariada de dados, a modelagem de equações estruturais baseada nos mínimos quadrados parciais - PLS-SEM. Esta técnica, além de permitir a análise simultânea de múltiplas variáveis, é indicada para ser trabalhada com amostras pequenas e por aceitar dados que não apresentem distribuição normal (Hair Junior, Jhult, Ringle, \& Sarstedt, 2016). Para tanto utilizou-se o software SmartPls 3.0.

Neste sentido, seguindo os pressupostos recomendados por Hair Junior et al. (2016), as 68 respostas válidas cumprem os requisitos mínimos, considerando o número de indicadores formativos e de caminhos estruturais em um único construto. Dessa forma, foi possível a realização das análises e conclusões apresentadas a seguir.

Em relação ao perfil das empresas presentes na amostra, 75\% estão inseridas em incubadoras de empresas, 42\% atuam na área de tecnologia da informação, $17 \%$ são do setor industrial, $16 \%$ da área de saúde, $12 \%$ atuam na área ambiental e $13 \%$ outras áreas. Com relação ao tempo de mercado, 59\% possuem menos de cinco anos de atuação, $28 \%$ possuem de cinco até dez anos de mercado e $13 \%$ estão há mais de dez anos no mercado. 


\section{Análise dos Resultados}

\subsection{Avaliação do Modelo de Mensuração - Modelo Formativo}

Avaliando a colinearidade entre os indicadores, os construtos formativos oportunismo e risco não apresentaram problemas de colinearidade. Dessa forma, iniciou-se a segunda etapa de avaliação, considerando a importância (significância) e relevância dos indicadores.

No que se refere à significância dos indicadores, ambos os construtos formativos não apresentaram indicadores significativos; porém, ao analisar a relevância dos indicadores, a maioria apresentou-se relevante, ou seja, com carga acima de 0,5 e significativos (Hair Junior et al., 2016). Apenas o indicador Q23 no construto Risco, e Q27 no construto Oportunismo não apresentaram relevância. Assim, pela validade de conteúdo optou-se por excluí-los do modelo.

\subsection{Modelo Reflexivo}

Ao analisar a confiabilidade dos indicadores, as cargas externas são observadas e, segundo Hair Junior et al. (2016), devem apresentar cargas iguais ou superiores a 0,708. Dessa forma, apenas um indicador do construto Confiança Interpessoal (Q8) apresentou carga abaixo de 0,4 e foi excluído do modelo. Outros dois indicadores Q15 e Q18 apresentaram cargas próximas de 0,7 e foram mantidos no modelo, considerando a validade conceitual.

Já em relação à consistência interna, todos os construtos apresentaram valores satisfatórios de confiabilidade composta (entre 0,884 e 0,925), bem como valores satisfatórios de Alfa de Cronbach (entre 0,824 e 0,877).

No que se refere à análise da validade convergente, analisando a variância média extraída (AVE), todos apresentaram valores acima do valor de referência de 0,5 (Hair Junior et al., 2016). Já na análise da validade discriminante pelo critério de Fornell-Larcker e pelo no teste de Heterotrait-monotrait (HTMT) das correlações, observou-se que os construtos Reputação, Competência, Boa Vontade e Capital Social não atenderam os requisitos mínimos para a validade discriminante. Dessa forma, optou-se por analisar a matriz de correlação dos indicadores pertencentes 
aos respectivos construtos. Pela análise da matriz de correlação e validade conceitual, optou-se por alterar o indicador Q5 do construto Boa Vontade para o construto Reputação, além de excluir os indicadores Q2 e Q3 do construto Competência, ficando este um construto de item único. A Figura 2 apresenta 0 modelo final testado.

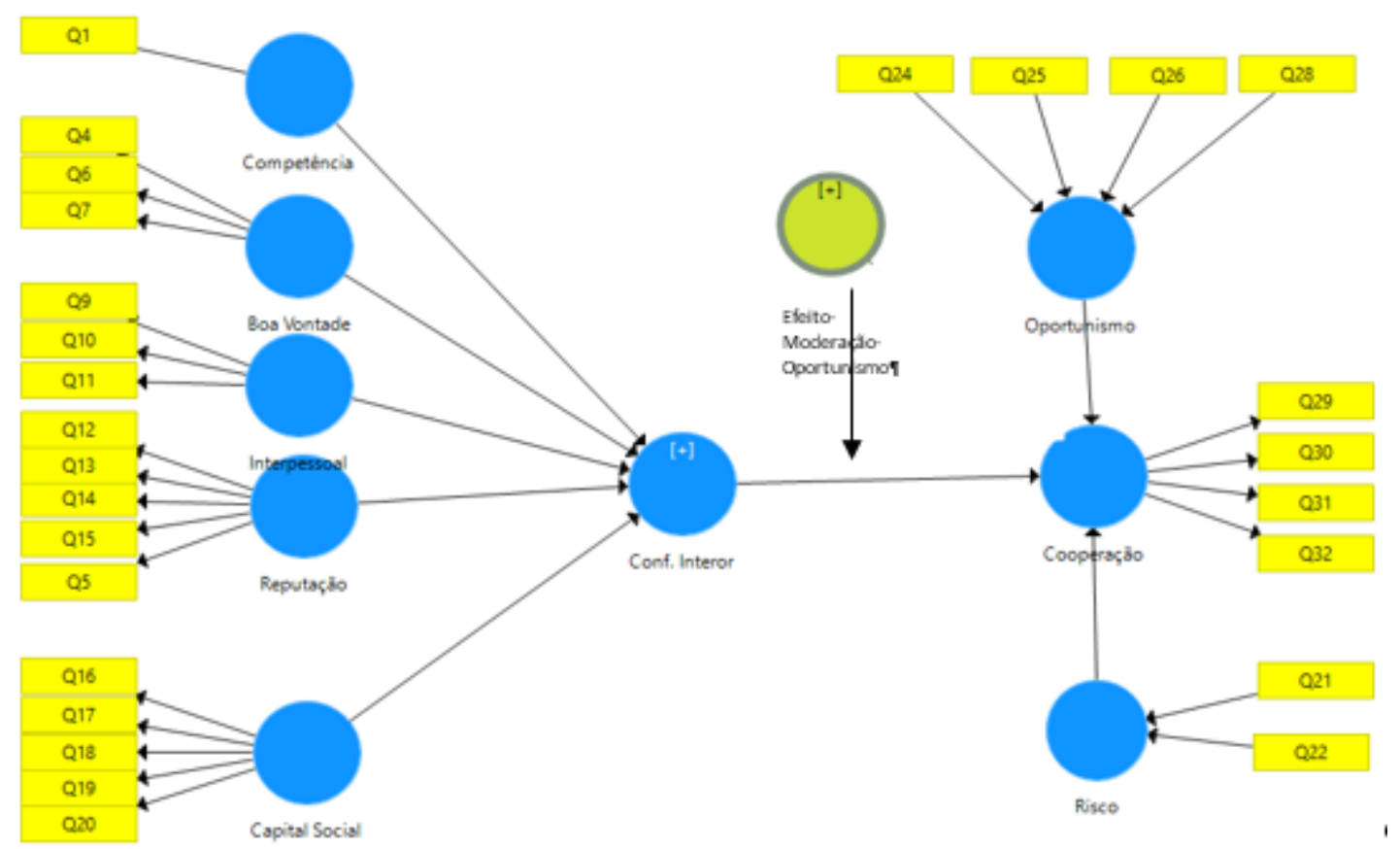

Figura 2

Modelo Final Testado

Fonte: Elaborado pelos autores.

Assim, após as alterações todos os testes foram considerados satisfatórios, passando então para a análise do modelo estrutural.

\subsection{Teste de Hipótese dos Coeficientes de Caminho}

Vale ressaltar que, para geração dos resultados utilizou-se a técnica do Bootstrapping com 5000 amostras, conforme recomenda Hair Junior et al. (2016).

\subsubsection{Formação da Confiança Interorganizacional}

No que se refere às hipóteses da formação da confiança (H1 até H5), Tabela 1 , todas as hipóteses foram significativas; assim, os resultados convergem com o 
esperado e indicado pela literatura, em que Competência, Boa Vontade, Confiança Interpessoal, Reputação e Capital Social contribuem para a formação da Confiança Interorganizacional.

A Reputação apresentou o maior coeficiente $(0,324)$, indicando que uma boa reputação favorece a formação da confiança entre empresas, o que vai ao encontro dos estudos de Das e Teng (1998). Corroborando, Houston (2003) afirma que a reputação positiva pode reduzir a assimetria de informação, fato que pode se refletir na relação da confiança.

A Competência na Confiança Interorganizacional foi o que apresentou menor coeficiente $(0,094)$, porém vale destacar que este construto passou a ser medido com apenas um indicador. Assim, pode não ter refletido de forma consistente o construto, mas apresenta uma significância de $1 \%$.

\section{Tabela 1}

Dados do Bootstrapping para teste de hipótese dos coeficientes de caminho

\begin{tabular}{|c|c|c|c|c|}
\hline & Coeficiente & $\begin{array}{l}\text { Estatística } \\
T\end{array}$ & $\begin{array}{l}\text { Valores } \\
\text { de } P\end{array}$ & \\
\hline H1: Competência -> C. Interorganizacional & 0,094 & 8,123 & 0,000 & $\begin{array}{l}\text { Não } \\
\text { Rejeita }\end{array}$ \\
\hline H2: B. Vontade -> C. Interorganizacional & 0,194 & 6,131 & 0,000 & $\begin{array}{l}\text { Não } \\
\text { Rejeita }\end{array}$ \\
\hline $\begin{array}{l}\text { H3: C. Interpessoal -> C. } \\
\text { Interorganizacional }\end{array}$ & 0,254 & 9,051 & 0,000 & $\begin{array}{l}\text { Não } \\
\text { Rejeita }\end{array}$ \\
\hline H4: Reputação -> C. Interorganizacional & 0,324 & 9,846 & 0,000 & $\begin{array}{l}\text { Não } \\
\text { Rejeita }\end{array}$ \\
\hline H5: C. Social -> C. Interorganizacional & 0,316 & 10,198 & 0,000 & $\begin{array}{l}\text { Não } \\
\text { Rejeita }\end{array}$ \\
\hline
\end{tabular}

Fonte: Elaborado pelos autores.

A Confiança Interpessoal formando a Confiança Interorganizacional foi confirmada a um nível de significância de 1\% e apresentando coeficiente de 0,254. Assim, o resultado também converge com o esperado, como indicaram Barney e Hansen (1994) e Tacconi et al. (2014) a Confiança Interorganizacional depende da Interpessoal, neste sentido a Confiança Interorganizacional perpassa a Interpessoal, demonstrando que a interação é importante no nível de aproximação. 
No que tange a Boa Vontade, esta apresentou um coeficiente de 0,194, significativo a 1\%, também confirmando a hipótese de que quando há intensão positiva esta contribui para a formação da confiança. Para Wang, Yeung e Zhang (2011) a presença da Boa Vontade gera uma cooperação mais efetiva e, portanto, um compartilhamento mais estreito de recursos o que aumenta o grau de comprometimento. Tal fato, pode então contribuir para a formação da confiança entre as empresas.

A hipótese H5: O Capital Social forma a Confiança Interorganizacional, também foi confirmada a um nível de significância de 1\% e com coeficiente de 0,316. O resultado demonstra a importância dos relacionamentos, no que se refere ao acesso de outros recursos, para a formação da Confiança.

\subsubsection{Impacto da Confiança na Cooperação}

Ao analisar o coeficiente de caminho entre os construtos Confiança Interorganizacional e Cooperação, conforme apresentado na Tabela 2, este apresenta um coeficiente de 0,881, com nível de significância de 1\%. Desse modo, a hipótese H6 "A Confiança Interorganizacional impacta a Cooperação" foi confirmada. Os resultados convergem com estudos anteriores como Gulati e Higgins (2003), Stuart (2000) e Thorgren e Wincent (2011), demonstrando que a Confiança é um fator determinante na Cooperação. Ou seja, com $99 \%$ de certeza os resultados demonstram que as empresas que pertencem aos habitats de inovação pesquisados, ao desenvolverem Confiança nas outras empresas tendem a desenvolverem práticas de Cooperação. Tal fato pode ocorrer diante de um alto nível de confiança, em que os parceiros permitem um compartilhamento mais efetivo de conhecimento e recursos; assim a cooperação se torna efetiva, conforme apontou Stuart (2000). 


\section{Tabela 2}

Dados do Bootstrapping para teste de hipótese dos coeficientes de caminho

$\begin{array}{lcccl} & \text { Coeficiente } & \text { Estatística T } & \text { Valores de } & \\ & & & P & \\ \text { H6:C. Interorganizacional -> } & 0,881 & 11,814 & 0,000 & \begin{array}{l}\text { Não } \\ \text { Cooperação } \\ \text { H7: Risco -> Cooperação }\end{array} \\ \begin{array}{lccc}\text { H8:Moderação oportunismo - } \\ \text { Cooperação }\end{array} & 0,157 & 1,849 & 0,065 & \begin{array}{l}\text { Não } \\ \text { Rejeita }\end{array} \\ & -0,328 & 2,846 & 0,004 & \text { Não } \\ \text { Rejeita }\end{array}$

Fonte: Elaborado pelos autores.

Para a hipótese $\mathrm{H} 7$ - O risco impacta a cooperação a partir da confiança estabelecida, foi encontrado um coeficiente caminho de 0,157 , com significância de $10 \%$. Portanto, pode-se inferir que a partir da incerteza as empresas tendem a desenvolverem práticas cooperativas. Dessa forma, não se rejeita a hipótese e como apontado por Hald, Cordon e Vollmann (2009) a incerteza é uma condição da confiança e, portanto, impacta na cooperação.

Por fim, os resultados referentes ao construto Oportunismo, revelam que, a hipótese H8 referente à moderação do Oportunismo na relação da Confiança e Cooperação apresentou um coeficiente negativo de 0,328 , com nível de significância de $1 \%$. Dessa forma, pode-se deduzir que o oportunismo possuiu um efeito na relação, porém um efeito negativo, como era esperado. Sendo assim, entende-se que comportamentos oportunistas tendem a reduzir a Cooperação a partir da Confiança. Tal resultado corrobora as demonstrações de Cabral (2008) de que o oportunismo traz um componente de incerteza na relação de confiança, impactando negativamente a Cooperação. $O$ tamanho do efeito de moderação será discutido no próximo tópico.

\subsection{Efeito Moderador}

Os resultados demonstraram que o efeito moderador do oportunismo na relação da Confiança com a Cooperação foi significativo; portanto, ao analisarmos o tamanho do efeito, observamos que a moderação tem um efeito negativo sobre a 
relação $(-0,027)$, enquanto o efeito simples da Confiança Interorganizacional sobre Cooperação é 0.881 . A Tabela 3 apresenta os resultados do efeito moderador na relação da Confiança com a Cooperação.

\section{Tabela 3}

Efeito Moderador

$\begin{array}{ll}\text { Efeito Simples } & \text { Tamanho } \\ \text { Moderação do Oportunismo } & 0,881 \\ \text { (Nível médio) } & -0,027 \\ \text { Oportunismo elevado* } & 0,854 \\ \text { Oportunismo baixo* } & 0,908\end{array}$

Fonte: Elaborado pelos autores.

* Considera-se o aumento ou diminuição a partir do nível médio em 1 desvio padrão.

Conjuntamente, esses resultados sugerem que a relação entre Confiança e Cooperação é de 0,881 para um nível médio de Oportunismo. Para níveis mais elevados de Oportunismo (por exemplo, aumentado por uma unidade de desvio padrão), a relação entre Confiança e Cooperação cai pelo tamanho do termo de interação (isto é, 0,881 - 0,027=0,854). Pelo contrário, para níveis mais baixos Oportunismo (por exemplo, diminuído por um ponto de desvio padrão), a relação entre Confiança e Cooperação torna-se maior $(0,881+0,027=0,908)$. Dessa forma, o efeito de moderação demonstrou um comportamento e tamanho de efeito conforme era esperado a partir da teoria, demonstrando que um comportamento de auto interesse prejudica a relação, deixando uma das partes mais vulnerável e, portanto, reduzindo a relação de Confiança e Cooperação (Cunha \& Melo, 2006; Das \& Teng, 1998; Mayer et al., 1995).

\subsection{Coeficiente de Determinação e Efeito Tamanho}

O coeficiente de determinação, ajustado do construto endógeno Cooperação, indica que $64 \%$ da variação da Cooperação é explicada pelas relações propostas. O efeito tamanho, segundo Hair Junior et al. (2016), é avaliado para determinar o impacto substancial de um construto omitido no construto endógeno, que neste caso é a Cooperação. Conforme observado na tabela 4, a Confiança possui um impacto alto no coeficiente de determinação da Cooperação, seguido por um efeito de 
tamanho médio pela moderação do Oportunismo e um efeito pequeno do risco sobre o $\mathrm{R}^{2}$ da Cooperação, o que reforça a importância do construto Confiança em relação às práticas cooperativas entre as empresas.

\section{Tabela 4}

Tamanho do Efeito f2

$\begin{array}{lll}\text { CONFIANÇA } & 1.588 & \text { Grande } \\ \text { INTERORGANIZACIONAL } & & \\ \text { MODERAÇÃO OPORTUNISMO } & 0.222 & \text { Médio } \\ \text { RISCO } & 0.046 & \text { Pequeno }\end{array}$

Fonte: Elaborado pelos autores.

\section{Considerações Finais}

Os resultados indicaram que a formação da confiança interorganizacional leva em consideração dimensões como a reputação, boa vontade, confiança interpessoal, competência e capital social (hipóteses H1, H2, H3, H4 e H5). Dessa forma, entende-se que, em ambientes de inovação, as empresas levam em consideração não só a credibilidade da organização parceira, quanto avaliam sua capacidade, bem como o acesso a novos recursos. No mesmo sentido, considerando que quem está à frente das organizações são as pessoas, a confiança interpessoal também contribui para que se forme a confiança interorganizacional. Da mesma forma a boa vontade, o que demonstrou, neste estudo, que a parte cognitiva também ajuda a formar a confiança.

Deste modo, a partir da formação da confiança pode-se perceber que esta impacta a cooperação em um contexto de risco e oportunismo. Assim, níveis de confiança elevados contribuem de fato para práticas cooperativas e espera-se que dessa forma haja impacto no processo de inovação, uma vez que, diante do modelo aberto de inovação, ocorre compartilhamento efetivo de informações e conhecimento (Melo, 2010). Neste caso, no que se refere às empresas pertencentes aos habitats de inovação, a relação confiança e cooperação se confirmou, sendo a variação na cooperação explicada por $64 \%$ da variação da confiança, risco e oportunismo. Portanto, torna-se essencial fomentar a formação da confiança entre as empresas, para que haja, cada vez mais, uma efetiva cooperação. 
Cabe destacar ainda, entre os resultados encontrados, que o oportunismo modera a relação de confiança e cooperação, ou seja, a relação é intensificada diante de comportamentos oportunistas que, quando presentes tendem a diminuir a relação.

Diante dos resultados encontrados, algumas implicações merecem ser destacadas: (i) identificou-se um forte impacto da confiança na cooperação; assim, para que a confiança seja formada é necessário que se criem relações, ou seja, que haja uma aproximação das empresas, para que questões como a confiança interpessoal seja desenvolvida. Além disso, compartilhar informações sobre os aspectos de competência e reputação das empresas também é essencial; (ii) as relações identificadas foram verificadas na presente amostra, portanto seria necessário verificar se o mesmo ocorre em outros contextos; (iii) diante dos resultados, e visando a desenvolver modelos abertos de inovação, tornam-se necessárias não apenas práticas para aproximação dos agentes de inovação, mas também buscar condições no que se refere a contextos econômicos e de incentivos para a formação de parcerias e redes de inovação, uma vez que as relações, neste caso, ocorrem fora do limite das organizações.

Conclui-se que os espaços relacionais de inovação (habitats) pesquisados possivelmente contribuem para a aproximação de agentes e, assim, cumprem seu papel no que tange à troca de conhecimento. Por fim, espera-se ter colaborado para uma reflexão acerca da temática confiança e cooperação discutida e sugere-se ainda que futuros estudos ampliem a amostra, inclusive para empresas que não pertencem a habitats de inovação, para que possa ser feita uma comparação. Além disso, é importante que novas pesquisas verifiquem o impacto no processo de inovação.

\section{Referências}

Abdalla, M. M., Calvosa, M. V. D., \& Batista, L. G. (2009). Hélice tríplice no Brasil: um ensaio teórico acerca dos benefícios da entrada da universidade nas parcerias estatais. Cadernos de Administração da Faculdade Salesiana Maria Auxiliadora, 1(3), 34-52.

Ali Babar, M., Verner, J.M., \& Nguyen, P.T. (2007). Establishing and maintaining trust in software outsourcing relationships: an empirical investigation. J. Syst. Softw, 80 (9), 1438-1449. 
Anand, V., Glick, W., \& Manz, C. (2002). Capital social: explorando a rede de relações da empresa. Revista de Administração de Empresas, 42(4), 57-71.

Barney, J. B., \& Hansen, M. H. (1994). Trustworthiness as a Source of Competitive Advantage. Strategic Management Journa, 15, 175-190.

Bhattacharya, R., Timothy M. D., \& Madan M. P.(1998). A formal model of trust based on outcomes. Academy of Management Review, 23, 459-472.

Brattstrom, A., Lofsten, H., \& Richtnér, A. (2012). Creativity, trust and systematic processes in product development. Research Policy, 41, 743- 755.

Britto, J. (2004). Cooperação e aprendizado em arranjos produtivos locais: em busca de um Referencial Analítico. Relatório de atividades do referencial conceitual, metodológico, analítico e propositivo - RedeSist. Rio de Janeiro: UFRJ.

Bukowitz, W. R., \& Williams, R. L. (2002). Manual de gestão do conhecimento: Ferramentas e técnicas que criam valor para a empresa. Porto Alegre: Bookman.

Cabral, R. M. (2008). Custos de Transação. In A. A. C. Callado, et al. Agronegócio (cap. 8, pp. 103-117). São Paulo: Atlas.

Chesbrough, H., Sohyeong, K., \& Agogino, A. (2014). Chez Panisse: building an open innovation ecosystem, California Management Review, 56(4), 144-171.

Chesbrough, H. (2003), Open Innovation: The New Imperative for Creating and Profiting from Technology. Harvard Business School Press.

Chesbrough, H. W. (2006). Open Innovation: A New Paradigm for Understanding Industrial Innovation. In H. Chesbrough, W. Vanhaverbeke, \& J. West (Eds.). Open Innovation: Researching a New Paradigm (pp. 1-12). Oxford: Oxford University Press.

Chesbrough, H. (2012). Inovação Aberta: Como criar e lucrar com a tecnologia. Porto Alegre: Bookman.

Cunha, C. R. da, \& Melo, M. C. D. O. L. (2006). A confiança nos relacionamentos interorganizacionais: o campo da biotecnologia em análise. RAE eletrônica, 5(2).

Cunha, J. A. C., Passador, J. L., \& Passador, C. S. (2012). A presença de agentes intermediadores na formação de redes interorganizacionais: uma análise sob a perspectiva temporal. Cadernos EBAPE.BR, 10(1), 108-128.

Czakon, W. (2009). Relational capability of organizations: theoretical advances. Journal of Economics \& Management, 5, 47-65.

Das, T. K., \& Teng, B. S. (1998). Between trust and control: Developing confidence in partner cooperation in alliances. Academy of Management Review 23(3), 491. 512.

Das, T. K., \& Teng, B. S. (2001). Trust, Control, and Risk in Strategic Alliances: an Integrated Framework. Organization Studies, 22(2), 251-283.

Del Campo, J. D. S. P. G., Pardo, I. P. G., \& Perlines, F. H. (2014). Influence factors of trust building in cooperation agreements. Journal of Business Research, 67(5), 710-714.

Diniz, B. A., \& Marconatto, D. A. (2010). Confiança: um construto universal. Revista de Administração FACES Journal, 10(1),. 35-55. 
Drechsler, W., \& Natter, M. (2012). Understanding a firm's openness decisions in innovation. Journal of Business Research, 65(3), 438-445.

Evans, J. R., \& Olsson, D. L. (2002). Introduction to simulation and risk analysis. New Jersey: Upper Saddle River.

Fink, M., Kessler, A., Duh, M., Belak, J., \& Lang, R. (2009). Trust and successful coordination on sme co-operation - an empirical study in Slovenia. Economic and Business Review, 11(3), 205-216.

Forcadell, F. J., \& Guadamillas, F. (2002). A case study on the implementation of a knowledge management strategy oriented to innovation. Knowledge and Process Management, 9(3), 162-171.

Freitas, H., Oliveira, M., Saccol, A. Z., \& Moscarola, J. (2000). O Método de Pesquisa Survey. Revista de Administração da USP-RAUSP, 35(3), 105-112.

Fryxell, G. E., Robert S. D., \& Vryza, M. (2002). After the ink dries: The interaction of trust and control in US-based international joint ventures. Journal of Management Studies, 39(6), 865-886.

Fukuyama, F. (1996). Confiança: as virtudes sociais e a criação da prosperidade (A. Lopes Trad.). Rio de Janeiro: Rocco.

Gulati, R., \& Higgins, M. C. (2003). Which ties matter when? The contingent effects of interorganizational partnerships on IPO success. Strategic Management Journal, 24(2), 127-144.

Hair Junior, J. F., Hult, G. T., M., Ringle, C., \& Sarstedt, M. (2016). A primer on partial least squares structural equation modeling (PLS-SEM). Sage Publications.

Hald, K. S., Cordon, C., \& Vollmann, T. E. (2009).Towards an understanding of attraction in buyer-supplier relationships. Industrial Marketing Management, 38(8), 960-970.

Hallin, C., Holm, U., \& Sharma, D. (2011). Embeddedness of innovation receivers in the multinational corporation: Effects on business performance. International Business Review, 20(3), 362-373.

Hao, M. A. (2004). Toward global competitive advantage creation, competition, cooperation, and cooption. Management Decision, New York, 42(7).

Houston, M. B. (2003). Alliance partner reputation as a signal to the market: Evidence from bank loan alliances. Corporate Reputation Review, 5(4), 330-342.

Jiang, X., Li, M., Gao, S. Bao, Y., \& Jiang, F.(2013). Managing knowledge leakage in strategic alliances: The effects of trust and formal contracts. Industrial Marketing Management, 42(6), 983-991.

Johnston, D. A., Mccutcheon, D. M., Stuart, F.I., \& Kerwood, H. (2004). Effects of supplier trust on performance of cooperative supplier relationships. Journal of Operations Management, 22(1), 23-38.

Laaksonen, T., Jarimo, T., \& Kulmala, H. I. (2009). Cooperative strategies in customer- supplier relationships: the role of interfirm trust. International Journal of Production Economics, 120(1), 79-87. 
Lavie, D., \& Drori, I. (2012). Collaborating for Knowledge Creation and Application: The Case of Nanotechnology Research Programs. Organization Science, 23(3), 704-724.

Lewis, J. D., \& Weigert, A. (1985). Trust as a social reality. Social Forces, 63,967985.

Luhmann, N. (2000). Familiarity, Confidence, Trust: Problems and Alternatives. In D. Gambetta. (Ed.) Trust: Making and Breaking Cooperative Relations. Electronic edition (Chapter 6, pp. 94-107). United Kingdom, Department of Sociology, University of Oxford.

Lui, S. S., \& Ngo, H. Y. (2004). The role of trust and contractual safeguards on cooperation in non-equity alliances. Journal of Management, 30(4), 471-485.

Luz, A. A., Kovaleski, J. L., Andrade Junior, P. P., Penteado, R. F. S., \& Zammar, A. (2014). Habitats de inovação e a sinergia do potencial acadêmico, tecnológico e inventivo em Ponta Grossa, Paraná, Brasil. Espacios, 35(6).

Machado, A. B., Silva, A. R. L., \& Catapan, A. H. (2016). Bibliometria Sobre Concepção de Habitats de Inovação. NAVUS - Revista de Gestão e Tecnologia, 6(3), 88-96.

Malhotra, D., \& Lumineau, F. (2001). Trust and collaboration in the aftermath of conflict: The effects of contract structure. Academy of Management Journal, 54(5), 981-998.

Mayer, R. C., Davis, J.H., \& Schoorman, F.D. (1995). An integrative model of organizational trust, Academy of Management Review, 20(3), 709-734.

Mcallister, D. J. (1995). Affect-and cognition-based trust as foundations for interpersonal cooperation in organizations. Academy of management journal, 38(1), 24-59.

Melo, H. S.(2010). Dicionário de tecnologia e inovação. Fortaleza: Sebrae.

Morgan, R. M., \& Hunt, S. D. (1994). The commitment-trust theory of relationship marketing. Journal of Marketing, 58(3), 20-38.

Nahapiet, J., \& Ghoshal, S. (1998). Social Capital, Intellectual Capital, and the Organizational Advantage. Academy of Management Review, 23(2), 242-266.

Newell, S., \& Swan, J. (2000). Trust and inter-organizational networking. Human Relations, 53(10), 1287-1328.

Nooteboom, B. (1996). Trust, opportunismand governance: A process and controlmodel. Organization Studies, 17(6), 985-1010.

Parashar, M. (2007). Steps to building innovating organizations. New Delhi: Response Books.

Porto, G. S. (2004). Características do processo decisório na cooperação EmpresaUniversidade. Revista de Administração Contemporânea, 8(3), 29-52.

RMI - Rede Mineira de Inovação. <www.rmi.org.br> Acessado em out/2016.

Rousseau, D.M., Sitkin, S.B., Burt, R.S., \& Camerer, C. (1998). Not so different after all: a cross- discipline view of trust. Academy of Management Review, 23(3), 393-404. 
Seppanen, R., Blomqvist, K., \& Sundqvist, S. (2007). Measuring inter-organizational trust: A critical review of the empirical research in 1990-2003. Industrial Marketing Management, 36(2), 249-265.

Shazi, R., Gillespie, N., \& Steen, J. (2015).Trust as a predictor of innovation network ties in project teams. International Journal of Project Management, 33(1), 81-91.

Sol, J., Beers, P. J., \& Wals, A. E. J. (2013). Social learning in regional innovation networks: Trust, commitment and reframing as emergent properties of interaction. Journal of Cleaner Production, 49, 35-43.

Stuart, T. E. (2000). Interorganizational alliances and the performance of firms: A study of growth and innovation rates in a high-technology industry. Strategic Management Journal, 21(8), 791-811.

Tacconi, M. de F. F. da S., Lopes, F. D., Mol, A. L. R., \& Tacconi Neto, E. A. (2014). A confiança interorganizacional nas compras. Gestão e Produção., São Carlos, 21(1), 199-214.

Thorgren, S., \& Wincent, J. (2011). Interorganizational Trust: Origins, Dysfunctions and Regulation of Rigidities. British Journal of Management, 22(1), 21-41.

Van Mierlo, B., Leeuwis, C., Smits, R., \& Woolthuis, R. K. (2010). Learning towards system innovation: Evaluating a systemic instrument. Technological Forecasting and Social Change, 77(2), 318-334.

Wang, L., Yeung, J. H. Y., \& Zhang, M. (2011). The impact of trust and contract on innovation performance: The moderating role of environmental uncertainty. International Journal of Production Economics, 134(1), 114-122.

Wittmann, C. M., Hunt, S. D., \& Arnett, D. B. (2009). Explaining alliance success: Competences, resources, relational factors, and resource-advantage theory. Industrial Marketing Management, 38(7), 743-756.

Zaheer, A., Mcevily, B., \& Perrone, V. (1998). Does Trust Matter? Exploring the Effects of Interorganizational and Interpersonal Trust on Performance. Organization Science, 9(2), 141-160.

Zahra, S. A. (2010). Harvesting Family Firm's Organizational Social Capital: A Relational Perspective. Journal of Management Studies, 47(2), 345-366.

Zarvandi, N., \& Zarvandi, J. (2012). Conceptualizations of Trust in the Organization. Ideal Type of Management,1(1), 37-46.

Zimmer, P., Luz Filho, S. S. da, \& Bloemer, R. (2015). Atributos geradores de confiança interorganizacional em redes de cooperação. Anais do $16^{\circ}$ Congresso Latino-lberoamericano de Gestão da Tecnologia, Porto Alegre, RS, Brasil.

\section{Apêndice}

\section{Tabela 1}

Construtos e variáveis do modelo. 


\begin{tabular}{|c|c|c|}
\hline Construto & Definição & Itens \\
\hline \multirow{3}{*}{ Competência } & \multirow{3}{*}{$\begin{array}{l}\text { Capacidade e habilidade do parceiro } \\
\text { de ter desempenho (Das \& Teng, } \\
\text { 2001; Nooteboom, 1996). }\end{array}$} & $\begin{array}{l}\text { Q1 - São capazes de realizar todas as } \\
\text { atividades propostas }\end{array}$ \\
\hline & & $\begin{array}{l}\text { Q2 - Possuem muito conhecimento sobre as } \\
\text { atividades desenvolvidas }\end{array}$ \\
\hline & & $\begin{array}{l}\text { Q3- Possuem habilidades importantes para } \\
\text { o negócio da minha empresa }\end{array}$ \\
\hline \multirow{4}{*}{ Boa vontade } & \multirow{4}{*}{$\begin{array}{l}\text { Crença na integridade das } \\
\text { organizações parceiras, ou seja, na } \\
\text { intenção positiva (Nooteboom, 1996; } \\
\text { Das \& Teng, 2001). }\end{array}$} & $\begin{array}{l}\text { Q4 - Desejam o melhor para a minha } \\
\text { organização, mesmo que isto não os } \\
\text { beneficiem diretamente }\end{array}$ \\
\hline & & Q5 - São honestas \\
\hline & & $\begin{array}{l}\text { Q6 - Ajudariam a minha organização em } \\
\text { momentos de dificuldades }\end{array}$ \\
\hline & & $\begin{array}{l}\text { Q7 - Se preocupam com as necessidades } \\
\text { da minha organização }\end{array}$ \\
\hline \multirow{4}{*}{$\begin{array}{l}\text { Confiança } \\
\text { Interpessoal }\end{array}$} & \multirow{4}{*}{$\begin{array}{l}\text { Confiança de um agente } \\
\text { organizacional que é depositada em } \\
\text { outro agente, dentro do limite } \\
\text { individual (Zaheer et al.,1998 }\end{array}$} & Q8 - Existe uma relação de amizade \\
\hline & & Q9 - Agem de forma ética \\
\hline & & Q10 - Agem de forma transparente \\
\hline & & $\begin{array}{l}\text { Q11 - São pessoas com habilidades } \\
\text { especializadas que contribuem para um } \\
\text { melhor desempenho }\end{array}$ \\
\hline
\end{tabular}

\begin{tabular}{|c|c|c|}
\hline \multirow{4}{*}{$\begin{array}{l}\text { Reputação } \\
\text { Organizacional }\end{array}$} & \multirow{4}{*}{$\begin{array}{l}\text { Integridade da organização parceira. } \\
\text { Relaciona-se com as características } \\
\text { comportamentais (Mcallister, 1995) }\end{array}$} & $\begin{array}{llll}\text { Q12 - Se comportam de forma } \\
\text { verdadeira }\end{array}$ \\
\hline & & Q13 - Cumprem com suas promessas \\
\hline & & $\begin{array}{l}\text { Q14 - Possuem um forte senso de } \\
\text { justiça }\end{array}$ \\
\hline & & $\begin{array}{llll}\text { Q15 - } & \text { Possuem } & \text { credibilidade } & \text { no } \\
\text { mercado } & & & \end{array}$ \\
\hline \multirow{5}{*}{ Capital Social } & \multirow{5}{*}{$\begin{array}{l}\text { Recursos que as organizações obtêm } \\
\text { por meio de suas relações com outras } \\
\text { organizações (Zahra, 2010) }\end{array}$} & $\begin{array}{l}\text { Q16 - Possuem acesso a outras } \\
\text { organizações importantes }\end{array}$ \\
\hline & & $\begin{array}{l}\text { Q17- Possuem acesso a recursos } \\
\text { importantes de outras organizações }\end{array}$ \\
\hline & & $\begin{array}{l}\text { Q18 - Se relacionam, na maioria das } \\
\text { vezes, com organizações que também } \\
\text { possuem credibilidade no mercado }\end{array}$ \\
\hline & & $\begin{array}{l}\text { Q19 - Contribuem para a geração de } \\
\text { novos conhecimentos }\end{array}$ \\
\hline & & $\begin{array}{l}\text { Q20 - Contribuem para a criação de } \\
\text { valor }\end{array}$ \\
\hline \multirow{4}{*}{ Risco } & \multirow{4}{*}{$\begin{array}{l}\text { Incerteza de resultados futuros (Evans } \\
\text { \& Olsson, 2002); probabilidade de } \\
\text { perda (Diniz \& MArconatto, 2010). }\end{array}$} & $\begin{array}{l}\text { Q21 - Existe muita incerteza relacionada } \\
\text { ao tipo de negócio (produto e/ou serviço) } \\
\text { que minha organização oferece }\end{array}$ \\
\hline & & Q22 - Existe perda elevada associada \\
\hline & & $\begin{array}{l}\text { ao compartilhamento do } \\
\text { desenvolvimento de produto ou serviço } \\
\text { que minha organização oferece }\end{array}$ \\
\hline & & $\begin{array}{l}\text { Q23 - O mercado de atuação da minha } \\
\text { organização é altamente fechado }\end{array}$ \\
\hline \multirow{2}{*}{ Oportunismo } & \multirow{2}{*}{$\begin{array}{l}\text { Relaciona-se na busca pelo auto } \\
\text { interesse (Mayer et al., 1995; Cunha } \\
\text { \& Melo, 2006; Das \&Teng, 1998) }\end{array}$} & $\begin{array}{l}\text { Q24 - Agem com comprometimento ao } \\
\text { que foi acordado }\end{array}$ \\
\hline & & $\begin{array}{l}\text { Q25 - São, na maioria das vezes, } \\
\text { imparciais em suas negociações }\end{array}$ \\
\hline
\end{tabular}




\begin{tabular}{|c|c|c|}
\hline & & $\begin{array}{l}\begin{array}{l}\text { Q26 - Cumprem todas as regras } \\
\text { contratuais }\end{array} \\
\end{array}$ \\
\hline & & $\begin{array}{l}\text { Q27 - Na maioria das vezes repassam } \\
\text { informações relevantes para meu } \\
\text { negócio. }\end{array}$ \\
\hline & & $\begin{array}{l}\text { Q28 - Agem de forma a não prejudicar a } \\
\text { minha organização }\end{array}$ \\
\hline \multirow{4}{*}{ Cooperação } & \multirow{4}{*}{$\begin{array}{l}\text { Iniciativa e a participação em acordos } \\
\text { colaborativos com outras } \\
\text { organizações; ação conjunta de } \\
\text { diferentes atores (Hao, 2004; Porto, } \\
2004)\end{array}$} & $\begin{array}{l}\text { Q29 - Minha organização, na maioria } \\
\text { das vezes, busca desenvolver acordos } \\
\text { colaborativos com outras organizações }\end{array}$ \\
\hline & & $\begin{array}{l}\text { Q30 - A partir das parcerias } \\
\text { desenvolvidas, na maioria das vezes, } \\
\text { surgem novas ideias de trabalhos } \\
\text { conjuntos }\end{array}$ \\
\hline & & $\begin{array}{l}\text { Q31 - Com parcerias minha organização } \\
\text { tem acesso a novas tecnologias durante } \\
\text { o desenvolvimento de projetos } \\
\text { conjuntamente }\end{array}$ \\
\hline & & $\begin{array}{l}\text { Q32 - A partir das parcerias } \\
\text { desenvolvidas minha organização, na } \\
\text { maioria das vezes, acumula aprendizado } \\
\text { e experiências }\end{array}$ \\
\hline
\end{tabular}

Fonte: Elaborado pelos autores a partir da revisão de literatura. 